\title{
PERSPECTIVA HISTÓRICA E ESTUDO DE CONCEITOS EM ECOLOGIA FUNCIONAL
}

\author{
Gabriel Antônio Rezende de Paula
}

Universidade Federal do Paraná, UFPR, Departamento de Zoologia, Programa de Pós-Graduação em Entomologia, Laboratório de Biologia Comparada de Hymenoptera, C.P. 19020, Curitiba, PR, Brasil, CEP: 81531-980. E-mail: gabrielrezp@gmail.com.br

\section{RESUMO}

Um dos objetivos centrais da ecologia é compreender as influências recíprocas entre a biodiversidade e o funcionamento do ecossistema. Por representarem a relação entre as funções dos organismos e os processos ambientais, as perspectivas funcionais podem refletir aspectos da organização e da dinâmica das comunidades biológicas não abrangidos pelas abordagens tradicionais. Tendo isso em vista, este estudo apresenta um levantamento bibliográfico sobre o surgimento e desenvolvimento de conceitos ecológicos relacionados à biologia das comunidades e a ecologia funcional, compreendendo uma discussão sobre os consensos adotados, intuitos da pesquisa e sua relação aos parâmetros baseados em agrupamentos taxonômicos. Observou-se que a síntese e a associação entre os fundamentos funcionais e taxonômicos podem compreender vias mais promissoras na realização de pesquisas voltadas ao estudo da dinâmica da biodiversidade, permanecendo necessários: um referencial teórico conciso e bem estabelecido, determinações precisas sobre os parâmetros adotados e a busca por práticas com fins de generalização que evidenciem padrões ecológicos.

Palavras-chave: Biodiversidade; grupo funcional; funcionamento do ecossistema; riqueza; abundância.

\section{ABSTRACT}

\section{HISTORICAL PERSPECTIVEAND STUDY OF CONCEPTS ON FUNCTIONALECOLOGY}

The reciprocal influences between biodiversity and ecosystem function comprise a central objective of ecology. By represent the relationship between the functions of the organisms and environmental processes, the functional perspectives might reveal aspects of the organization and dynamics of biological communities unsolved by traditional approaches. About these points, this study presents a literature research over the emergence and development of general ecological concepts related to biology of communities and functional ecology, including a discussion of the adopted consensus, aims of study and their association with the traditional parameters of research. It was noted that the synthesis and association of taxonomic and functional perspectives may comprise the most promising areas on realization of studies of biodiversity dynamics. However, is still necessary: a well established and concise theoretical support, accurate determinations of adopted parameters and the search for practices for uncover ecological patterns with purposes of generalization.

Key words: Biodiversity, functional group, ecosystem function, richness, abundance.

\section{INTRODUÇÃO}

A importância da diversidade de espécies para o funcionamento do ecossistema tem emergido como uma questão chave na biologia de conservação (Hochberg and Hawkins 1992, Dalerum et al. 2012), existindo evidências substanciais sobre a influência mútua entre esses dois fatores (Flynn et al. 2011). Isso se deve ao fato de o funcionamento do ecossistema compreender a dinâmica temporal dos processos ambientais, como a produção primária, a transferência trófica entre plantas e animais, a ciclagem de nutrientes e a transferência de calor, abrangendo as taxas e níveis de concentração, ganho ou perda de energia e materiais através dos componentes abiótico e biótico (Díaz and Cabido 2001, Tilman 2001, van der Linden et al. 2012).

Há décadas realizam-se pesquisas sobre a conceituação e aplicação da diversidade biológica, as quais têm possibilitado o conhecimento sobre o seu valor intrínseco, além do levantamento de hipóteses que abrangem o estudo sobre a riqueza das espécies e suas variações no tempo e no espaço (Barbault 1995, Purvis and Hector 2000). Tais hipóteses são essenciais para os estudos evolutivos, agroflorestais, biotecnológicos e farmacêuticos (Barbault 1995). Contudo, grande parte do crescente interesse na diversidade biológica e de sua relação com as funções do ecossistema é resultante da preocupação com a preservação da biosfera, da riqueza de espécies e dos serviços ambientais por elas fornecidos (Duarte 2000, Purvis and Hector 2000, Schwartz et al. 2000, Cameron 2002).

Esses serviços compreendem os produtos e processos presentes no ambiente, dos quais os seres humanos se beneficiam (Díaz and Cabido 2001), abrangendo o conjunto de atividades biogeoquímicas derivadas das interações físicas e químicas dos 
organismos entre si ou com ambiente, tendo-se como exemplos: a produtividade (alimentos, fibras, combustíveis, etc.), a disponibilidade de água, o sequestro de carbono, a decomposição e a ciclagem e retenção de nutrientes, a formação de solo, a desintoxicação, a recreação e o melhoramento do clima (Cardinale et al. 2000, Duarte 2000, Schwartz et al. 2000, Díaz and Cabido 2001, Naeem and Wright 2003, Zayed et al. 2005). Funções frequentemente compartilhadas entre os organismos, parecendo ser amplamente dependentes de um número representativo de espécies abundantes (Duarte 2000); de modo que o elo entre a biodiversidade, os serviços ambientais e o funcionamento do ecossistema possui natureza complexa (Cameron 2002).

A necessidade de um monitoramento das respostas da biodiversidade aos distúrbios e de um entendimento sobre como isto interfere na funcionalidade dos ecossistemas (Podgaiski et al. 2011) proporcionou a formação de um conjunto de estudos que buscam descrever a importância das influências recíprocas entre a riqueza de espécies e as funções ecológicas (Cameron 2002, Duff 2003, Naeem and Wright 2003), com o subsequente aumento em experimentos empíricos de quantificação da diversidade funcional (Dalerum et al. 2012, Díaz and Cabido 2001). Desse modo, o papel da riqueza de espécies não tem sido analisado isoladamente (Dalerum et al. 2012), uma vez que padrões ecológicos significativos podem ser mascarados ao se considerar como equivalentes organismos que apresentam uma ampla variação em seus atributos biológicos (Grönroos and Heino 2012).

A diversidade funcional compreende um dos componentes da diversidade biológica (Tilman 2001) e surgiu como uma ferramenta que vai além dos conceitos tradicionais por analisar os vários modos como os organismos respondem à variabilidade ambiental (Gerisch et al. 2012). Ela corresponde a uma medida da amplitude, dispersão ou abundância relativa dos atributos das espécies ou organismos (Díaz and Cabido 2001, Tilman 2001, Gerisch et al. 2012), abrangendo uma mensuração da diferenciação ecológica entre hábitos ou entre funções e podendo refletir os componentes mais relevantes associados aos padrões na comunidade (Gitay and Noble 1997, Thompson et al. 2010, de Bello 2012).

A diversidade funcional é aferida para se determinar como a composição funcional varia dentre as espécies no tempo e no espaço, além de buscar-se uma melhor compreensão de como tal variação influencia o funcionamento do ecossistema (Petchey and Gaston 2007). Dessa forma, medir a diversidade funcional significa medir a diversidade de características fenotípicas dos organismos que influenciam os processos na comunidade (Cianciruso et al. 2009). Tais estudos, mesmo em início de discussão, já resultaram em um crescente escopo teórico e metodológico, em modelos quantitativos e observações de campo (Schwartz et al. 2000, Díaz and Cabido 2001, Gerisch et al. 2012) que têm demonstrado que as comunidades regulam os processos ecológicos, de modo que se busca compreender a natureza desse fenômeno através de respostas a duas questões fundamentais: a) Qual a natureza da relação entre a diversidade de espécies e o funcionamento do ecossistema? e, b) tal relação é mais influenciada pela riqueza ou pelos tipos particulares de espécies? (Cardinale et al. 2000, Naeem and Wright 2003).

A princípio, evidências empíricas baseadas na diferenciação de nichos, conduziram ao consenso de que a riqueza de espécies era a contribuinte primária para o funcionamento do ecossistema (Cardinale et al. 2000, Díaz and Cabido 2001). Contudo, atualmente há o consenso de que a estrutura da assembleia pode ser tão importante quanto sua riqueza (Naeem and Wright 2003, Dalerum et al. 2012), uma vez que nem todas as espécies são equivalentes em seu papel no ecossistema, e os processos ecológicos podem ser amplamente influenciados por tipos particulares ou pelo modo de combinação das espécies presentes (Cardinale et al. 2000).

A ausência inicial de evidências sobre a importância das particularidades das espécies pode ser devida aos estudos terem se iniciado apenas no começo dos anos 90, com sua maioria lidando indiretamente com a diversidade funcional (Díaz and Cabido 2001, Naeem and Wright 2003), através de componentes mais fáceis de estimar, a exemplo de índices (Nilman 2001). Além disso, observa-se inconsistência nos conceitos e filosofia sobre a funcionalidade ambiental, de modo que a atribuição funcional à diversidade biológica permanece questionável (Barbault 1995).

Portanto, esta revisão compreende um levantamento bibliográfico sobre o surgimento e o desenvolvimento de conceitos em ecologia das comunidades relacionados às dinâmicas funcionais, abrangendo uma discussão sobre os consensos 
adotados e perspectivas de estudo. Busca-se apresentar a evolução do suporte teórico dessa área de conhecimento e os caminhos de contraposição ou associação aos parâmetros tradicionais.

\section{RELAÇÕES ENTRE BIODIVERSIDADE, BIOLOGIA DE COMUNIDADES E ECOLOGIA FUNCIONAL}

O termo biodiversidade, contração de diversidade biológica, como conceito clássico na ecologia, se baseia no reconhecimento da riqueza e da diversidade de espécies. Compreende uma medida da variedade, número ou multiplicidade de táxons em uma área restrita ou em uma região, usualmente contemplando a variação gênica, fenotípica, a unicidade taxonômica e o endemismo, além das fitofisionomias em determinado sistema (Barbault 1995, Cardinale et al. 2000, Purvis and Hector 2000, Díaz and Cabido 2001, Tilman 2001).

A estimativa da biodiversidade possibilita o conhecimento sobre a distribuição dos organismos (Purvis and Hector 2000, Brooks et al. 2012), consistindo em um conceito multidimensional que não pode ser reduzido a um valor exclusivo incapaz de representar a variedade das formas de vida. Desse modo, a biodiversidade é usualmente estudada de forma integrada, por meio de medidas de diferentes perspectivas quantificadas através dos distintos níveis de organização dos sistemas biológicos e ecológicos comparáveis dentre regiões ou momentos históricos (Purvis and Hector 2000).

Tais perspectivas correspondem a uma reunião de princípios relacionados. Como exemplo, a biodiversidade pode ser definida em três níveis: a) diversidades genética, intraespecífica e fenotípica, ao nível de espécies e populações; b) diversidade de espécies; e c) diversidade do ecossistema, ecológica ou funcional, ao nível das redes tróficas e paisagens (Barbault 1995, Purvis and Hector 2000).

Contudo, permanece em debate qual dessas facetas possui maior influência sobre as funções ecológicas (Flynn et al. 2011), sendo recorrente a premissa de que as características das espécies influenciam sua distribuição, tendo participação na construção de uma comunidade. Desse modo, o estudo da biodiversidade tem relação direta com os de ecologia de comunidades, os quais consideram a estrutura hierárquica e a funcionalidade dos organismos que a compõem, relacionando padrões e processos e dando ênfase aos mecanismos que conduzem a permanência, elevação ou redução das populações (Barbault 1995).

Tais princípios são observados desde a conceituação clássica de comunidades como superorganismos, de Clements (Clements 1916); passando pelo conceito individualista dos conjuntos livres, de Gleason (Gleason 1939); ou pela perspectiva oposta e integrativa de Whittaker, em que as espécies seriam reunidas pela imposição de forças direcionais de poder suficiente para compor uma unidade que compreenderia a comunidade (Whittaker 1951, Barbault 1995). Além disso, algumas das ideias iniciais sobre como a biodiversidade pode afetar o funcionamento do ecossistema são atribuídas a Darwin e Wallace, os quais afirmaram que composições diversas de plantas poderiam ser mais produtivas do que monoculturas, sugerindo o conceito de nichos ecológicos e o princípio geral de que comunidades mais diversas e intactas são geralmente mais estáveis e funcionalmente melhores (Purvis and Hector 2000, Tilman 2001, Violle et al. 2007).

Entretanto, os estudos de organização das comunidades ganharam impulso significativo apenas durante os anos 60 e o início da década de 70 , sob o ímpeto de Hutchinson e MacArthur, quando a atenção foi direcionada às interações bióticas, em especial à competição interespecífica. Na década de 80 , a competição tornou-se apenas um dos fatores de análise e as variabilidades espacial e temporal surgiram como pontos de importante atenção (Barbault 1995). Posteriormente, as pesquisas passaram a ser conduzidas na busca de uma melhor compreensão de como as espécies interagem, de sua distribuição e da dinâmica de suas respostas às variações ambientais. $\mathrm{O}$ que foi suportado pelo conhecimento de que a diversidade biológica e os processos biogeoquímicos que caracterizam o ecossistema representam o saldo da história evolutiva e da parcela extrínseca de mudanças nos fatores abióticos, (Bronw and Heske 1990, Naeem and Wright 2003).

\section{O SURGIMENTO DOS CONCEITOS E PERSPECTIVAS EM ECOLOGIA FUNCIONAL}

As características funcionais em uma comunidade representam as interações que interferem na abundância e dinâmica populacional dos organismos, nas estruturas trófica e ambiental (Naeem and Wright 2003). Desta forma, em ecologia, as palavras função, funcional e funcionamento se referem ao modo pelo 
qual o ecossistema opera, sem condicionar um propósito ou um objetivo, o que seria inconsistente aos preceitos evolutivos (Tilman 2001).

Os estudos de relação entre a biodiversidade e o funcionamento do ecossistema se iniciaram no começo dos anos 90, quando a maioria dos trabalhos empregaram análises combinatórias destinadas à manipulação da diversidade taxonômica para representar conjuntos funcionais encontrados no ambiente em investigação (Naeem and Wright 2003). Já ao fim da mesma década, os ecólogos deram maior ênfase ao uso de classificações não filogenéticas dos organismos, sendo que alguns consideravam que a taxonomia clássica iria ceder lugar às classificações funcionais na descrição da estrutura e funcionamento do ecossistema (Gitay and Noble 1997, Gitay et al. 1999).

Contudo, observa-se que a ideia de grupos funcionais surgiu por várias vezes na história dos conceitos ecológicos (Barbault 1995). Classes funcionais em plantas já haviam sido desenvolvidas por naturalistas e fisiólogos, e as classificações por características de funcionalidade podem ser pontuadas desde Theophratus, a cerca de 300 a. C. (Barbault 1995, Gitay and Noble 1997). Posteriormente, as descrições de Kölreuter's, em 1761, e de Sprengel's, em 1793, indicaram que os polinizadores poderiam ser reunidos em grupos de organismos de comportamento similar que exerciam pressões seletivas parecidas sobre as flores, compreendendo um importante passo para os estudos de síndromes de polinização. Em 1862, Darwin e outros estudiosos elaboraram a perspectiva de que combinações de características florais poderiam refletir o tipo de polinizador associado (Fenster et al. 2004). E em 1934, Raunkiaer indicou que agrupamentos de formas de vida de plantas eram úteis em análises sobre a influência do clima na composição e dinâmica vegetais, prática amplamente aceita por ser fundamentada em critérios morfológicos generalizados para todas as espécies (Barbault 1995).

Contudo, como aspectos importantes da vegetação não são expressos apenas pelas referências morfológicas, tornou-se necessário o reconhecimento de características adicionais, as quais poderiam ser relacionadas ao hábitat e ecologia vegetais (Barbault 1995). Tal perspectiva originou inconsistência na terminologia e o surgimento de muitos termos que têm sido utilizados para se expressar o mesmo princípio, a exemplos de formas de crescimento, formas de vida e estratégias
(Semenova and van der Maarel 2000). A construção moderna do conceito iniciou-se apenas com sugestões levantadas nos anos 60, dentre os ecólogos que, seguindo Hutchinson e MacArthur, adotaram o consenso sobre a organização das comunidades em assembleias de espécies definidas por sua base funcional (Barbault 1995, Gitay and Noble 1997).

Em 1967, Root introduziu o termo guilda, definindo-o como um grupo de espécies que exploram de modo similar uma classe de recursos ambientais, ou um conjunto de espécies que se sobrepõem significantemente em seus requerimentos de nicho (Barbault 1995, Gitay and Noble 1997). Sob tal perspectiva, espécies pertencentes a um mesmo guilda são mais propensas a apresentar fortes interações entre si, enquanto que diferentes guildas podem possuir distintos requerimentos ambientais, devido aos respectivos hábitos alimentares e fontes de recursos (Grönroos and Heino 2012).

O conceito foi também fundamentado no desenvolvimento e definição dos níveis tróficos (conjunto de táxons que compartilham um hábito alimentar) em trabalhos de pesquisadores teóricos interessados na estrutura e complexidade de redes alimentares (Barbault 1995). Tais estudiosos denominaram espécies tróficas como o conjunto de organismos que ocupam primordialmente o mesmo local na rede alimentar, se nutrindo de uma fonte de recursos comum, e compreendendo também uma base alimentar para outro grupo (Briand and Cohen 1987, Barbault 1995).

No primeiro capítulo do livro Plant Functional Types - Their Relevance for Ecosystem Properties and Global Change, denominado What are functional types and how should we seek then?, Gitay and Noble (1997) apresentam um breve histórico relacionado à conceituação de tipos funcionais (demonstrado de forma resumida na Tabela 1). Os autores argumentam que do final da década de 80 ao início dos anos 90, grupos funcionais correspondiam usualmente aos componentes do ecossistema que realizam a mesma função, ou conjunto de funções. Os termos tipos funcionais e grupos funcionais seriam sinônimos, apresentando um conjunto de ideias similares às diversas definições de guildas, se distinguindo por serem agrupamentos determinados pela similaridade nos mecanismos de respostas às variações ambientais, enquanto que nos guildas o conjunto de espécies é reunido devido ao uso comum de recursos. 
Estudos têm demonstrado que dificilmente será possível estabelecer-se uma classificação universal para os tipos funcionais ou para os outros conceitos relacionados (Skarpe 1996, Semenova and van der Maarel 2000, Violle et al. 2007). Isso se deve ao fato de que não é simples ter-se uma definição precisa para o termo, devido à ampla faixa de modos em que o preceito tem sido e provavelmente permanecerá sendo utilizado (Gitay and Noble 1997, Gitay et al. 1999), de modo que o perfil adotado deve ser esclarecido e apresentado em acordo aos diferentes propósitos e níveis de resolução em que é empregado (Skarpe 1996).

Como exemplo, o termo pode ser delimitado a agrupamentos de organismos baseados em suas respostas às perturbações, podendo reunir diversos níveis taxonômicos ou diferentes estágios de desenvolvimento que apresentam um conjunto comum de características previamente selecionadas pelo pesquisador (Gitay and Noble 1997, Naeem and Wright 2007). Enquanto Díaz and Cabido (2001) abrangem os tipos funcionais à perspectiva de reunião de organismos que compartilham respostas similares ao ambiente (temperatura, disponibilidade de água e nutrientes, regimes de fogo e geadas, dentre outros), além de efeitos parecidos sobre o funcionamento do ecossistema (por exemplo, quanto à produtividade, ciclagem de nutrientes e resiliência). Confluindo ao conceito de Tilman (2001), pela percepção que integra as respostas e as ações dos organismos no ambiente.

Desse modo, orientando-se pela literatura e em observação à necessidade de uma conceituação frequente, inequívoca, descritiva e sintética (Violle et al. 2007), um grupo funcional poderia ser precisamente definido como: o agrupamento de organismos em uma classificação não filogenética, o qual abrange os indivíduos, ou espécies, que compartilham uma série de atributos de história de vida, ou atributos funcionais, e que se relacionam (em resposta e efeito) de modo similar a uma síndrome de fatores ambientais.

Tabela 1. Resumo histórico de conceitos referentes aos grupos funcionais, baseados em Briand and Cohen (1987), Gitay and Noble (1997) e Tilman (2001). Historical summary of concepts related to functional groups, based on Briandand Cohen (1987), Gitay and Noble (1997) e Tilman (2001).

\begin{tabular}{|c|c|c|c|c|}
\hline ANO & AUTORES & TERMO & CONCEITO & RELAÇÃo \\
\hline 1974 & Cummis & grupo funcional & $\begin{array}{l}\text { Conjunto de organismos através dos quais se podem } \\
\text { expressar importantes aspectos de processos ecológicos. }\end{array}$ & - \\
\hline 1975 & Botkin & tipos funcionais & $\begin{array}{l}\text { Reunião de um número mínimo de espécies em grupos } \\
\text { através dos quais as espécies fornecem informações sobre } \\
\text { interações importantes na população. }\end{array}$ & - \\
\hline 1980 & Paine & módulo & Grupo de espécies interagindo intimamente. & - \\
\hline 1982 & Yodzis & clique & $\begin{array}{l}\text { Conjunto de espécies que resguardam um recurso alimentar } \\
\text { em comum (não necessariamente o utilizando do mesmo } \\
\text { modo), sendo o clique dominante um clique que não está } \\
\text { contido em nenhum outro, podendo também ser } \\
\text { denominado de guilda trófica. }\end{array}$ & - \\
\hline 1982 & Bahr & $\begin{array}{l}\text { grupos } \\
\text { ecológicos de } \\
\text { espécies }\end{array}$ & $\begin{array}{l}\text { Grupo de espécies que exploram a mesma classe de recursos } \\
\text { ambientais de modo similar. }\end{array}$ & - \\
\hline 1981 & Jaksic & $\begin{array}{c}\text { guildas } \\
\text { operacionais }\end{array}$ & $\begin{array}{l}\text { Grupo de espécies que respondem de modo similar a uma } \\
\text { variedade de mudanças propensas a afetar seu ambiente. }\end{array}$ & \multirow{3}{*}{$\begin{array}{l}\text { Divisões ou fusões do } \\
\text { conceito básico de } \\
\text { guildas. }\end{array}$} \\
\hline 1986 & Briand e Cohen & espécies tróficas & $\begin{array}{l}\text { Conjunto de organismos que se alimentam em um conjunto } \\
\text { comum de seres vivos e que por sua vez, também } \\
\text { compreendem uma fonte alimentar comum para outro } \\
\text { determinado grupo. }\end{array}$ & \\
\hline 1986 & Szaro & guilda funcional & $\begin{array}{l}\text { Mesmo significado essencial do termo de Root, como } \\
\text { apresentado no texto: Grupo de espécies que exploram a } \\
\text { mesma classe de recursos ambientais de modo similar. } \\
\text { Adicionando mais dois termos: } \\
\text { a) guildas estruturais - grupo de espécies que usa o mesmo } \\
\text { recurso, não necessariamente do mesmo modo ou com o } \\
\text { mesmo propósito; e b) guildas de resposta - espécies que } \\
\text { respondem de modo similar à perturbação ambiental. }\end{array}$ & \\
\hline
\end{tabular}




\begin{tabular}{|c|c|c|c|c|}
\hline ANO & AUTORES & TERMO & CONCEITO & RELAÇÃo \\
\hline 1986 & $\begin{array}{l}\text { Menge, } \\
\text { Lubchenco, } \\
\text { Ashkenas e } \\
\text { Ramsey }\end{array}$ & guilda funcional & $\begin{array}{l}\text { Apresentaram a mesma dicotomia descrita por Szaro, sendo } \\
\text { que as guildas de resposta foram nomeados de tipos } \\
\text { funcionais. }\end{array}$ & \\
\hline 1988 & Grime & estratégia & $\begin{array}{l}\text { Grupo de características genéticas análogas ou similares, } \\
\text { freqüentes entre as espécies ou populações, às quais } \\
\text { condicionam ecologia similar. }\end{array}$ & - \\
\hline 1991 & Faber & liga & $\begin{array}{l}\text { Conjunto de organismos que utilizam mais de um recurso } \\
\text { de modo similar. }\end{array}$ & - \\
\hline 1991 & Barbault & tipos funcionais & $\begin{array}{l}\text { Grupos de organismos definidos em termos de morfologia e } \\
\text { fisiologia, particularmente por estas características estarem } \\
\text { associadas aos recursos e interações entre espécies, } \\
\text { representando guildas alimentares ou modos de } \\
\text { desenvolvimento vegetal. }\end{array}$ & - \\
\hline 1988 & $\begin{array}{l}\text { Fridel, Bastin e } \\
\text { Griffin }\end{array}$ & grupo funcional & Grupos que respondem similarmente à mesma perturbação. & \multirow{3}{*}{$\begin{array}{l}\text { Componentes do } \\
\text { ecossistema que } \\
\text { realizam a mesma } \\
\text { função, ou o mesmo } \\
\text { conjunto de funções. }\end{array}$} \\
\hline 1989 & Noble & grupo funcional & $\begin{array}{l}\text { Classificação baseada nos atributos fisiológicos, } \\
\text { reprodutivos e caracteres de história de vida, onde a } \\
\text { variação em cada caráter tem um valor ecológico preditivo } \\
\text { específico (mais do que descritivo). }\end{array}$ & \\
\hline 1992 & Keddy & grupo funcional & $\begin{array}{l}\text { Grupos de espécies que compartilham características } \\
\text { similares. }\end{array}$ & \\
\hline 2001 & Tilman & grupo funcional & $\begin{array}{l}\text { Conjunto de Espécies que possuem características similares } \\
\text { e que, desse modo, são propensas por apresentarem efeitos } \\
\text { semelhantes sobre o funcionamento do ecossistema. }\end{array}$ & - \\
\hline
\end{tabular}

\section{ASSOCIAÇÃO ENTRE ECOLOGIA FUNCIONAL E A DINÂMICA DA BIODIVERSIDADE}

Abordagens baseadas em atributos são amplamente utilizadas em estudos de ecologia e evolução, e dentre as diferentes áreas de pesquisa diversos tipos de atributos têm sido utilizados para revelar componentes inerentes a processos complexos. Contudo, síncrono à emergência da ecologia funcional como disciplina, surgiu o termo atributo funcional, visto como a pedra fundamental na reformulação da ecologia de comunidades. (Violle et al. 2007).

Um atributo funcional corresponde a um determinado estado ou modalidade de um caráter ou característica fenotípica (morfológica, fenológica, comportamental ou fisiológica), mensurável a partir do nível celular ao do organismo como um todo, e que está associado a um processo biogeoquímico ou a uma propriedade do ecossistema em investigação (Naeem \& Wright 2007, Violle et al. 2007, Podgaiski et al. 2011), sendo relevante para a resposta do organismo ao ambiente ou para os seus efeitos sobre os processos ecológicos (Díaz \& Cabido 2001). A distribuição de um atributo funcional não é necessariamente dependente da existência de ancestrais comuns, mas também não está randomicamente dispersa na história evolutiva de um grupo (Naeem \& Wright 2003, Bruno \& Olivier 2007). Por outro lado, algumas características podem ser exclusivas de uma espécie ou expressas por poucas, ou podem estar presentes apenas em determinado contexto ambiental ou estágio do ciclo de vida de um organismo (Naeem \& Wright 2003, Bruno \& Olivier 2007), uma vez que os atributos podem variar dentre as espécies ao longo de gradientes ambientais ou temporais, não havendo uma característica exclusiva (Violle et al. 2007).

Tais propriedades também se relacionam à possibilidade de considerar as variações intraespecíficas (Podgaiski et al. 2011), uma vez que a inclusão das diferenças funcionais entre indivíduos podem ser importantes para os processos da comunidade quando influenciam a habilidade competitiva, a coexistência, a ciclagem de nutrientes, 
a resistência a perturbações e a produtividade dos indivíduos de uma espécie (Cianciaruso et al. 2009). Como exemplo, Bruno \& Olivier (2007) apresentam estudos que têm quantificado o caráter adaptativo pelo uso de atributos morfológicos para as comunidades de plantas aferindo a variabilidade intraespecífica.

Violle et al. (2007) enfatizam que um atributo não deveria abranger qualquer elemento externo aos indivíduos (parâmetros demográficos ou fatores ambientais) ou qualquer outro nível de organização biológica (propriedades da população, comunidade ou ecossistema, a exemplo da biomassa acumulada). Contudo, a percepção de características funcionais relacionadas a um dado fenômeno ambiental é variável e dependente de numerosos fatores (Naeem \& Wright 2003), de modo que o conceito tem sido amplamente utilizado em níveis diversos de organização. Quanto à natureza dos dados, os atributos podem ser de diferentes tipos: quantitativos contínuos (como exemplo: comprimento e largura de estruturas morfológicas, peso, taxa metabólica), discretos (idade, número de ovos, etc.), binários (indicando a presença ou ausência de determinado atributo) ou categóricos (cores, sexo ou casta, dentre outros) (Podgaiski et al. 2011).

Os atributos podem interagir de modo não linear, sinérgico, antagônico, ou em tantas outras maneiras que a combinação de diferentes características pode ser mais determinante para a distribuição das espécies do que sua simples associação (Bruno and Olivier 2007, Rocha et al. 2011). Conjuntamente, numerosos fatores abióticos - como a temperatura, condições do solo, umidade, precipitação, disponibilidade de nutrientes ou tempo decorrente desde o distúrbio - interferem na composição, distribuição e abundância das espécies (Naeem and Wright 2003), influenciando na interpretação das influências das características funcionais em distintas condições ambientais (Rocha et al. 2011).

Contudo, há suporte teórico e empírico sobre a existência de uma forte relação entre os atributos funcionais das espécies e suas respostas às variáveis ambientais (Rocha et al. 2011). Cada espécie possui um elevado número de características que se relacionam ao papel que elas possuem no ambiente, podendo interferir em sua abundância e nas funções ecossistêmicas (Tilman 2001, Brooks et al. 2012). Tal relação é proveniente do fato do desempenho de uma espécie ser dado pela sua habilidade em crescer, reproduzir ou sobreviver, o fitness darwiniano, que pode ser representado e é influenciado (direta ou indiretamente) por atributos como o tamanho do corpo e a maturação, dentre outros (Violle et al. 2007).

Uma vez que os atributos funcionais podem capturar dimensões chave da biodiversidade de uma maneira simplificada, abrangente e preditiva (Podgaiski et al. 2011), eles têm sido incorporados em modelos das funções ecossistêmicas com o intuito de refletir alterações pontuais na comunidade e na composição de guildas (De Bello et al. 2010, Willians et al. 2010, Rocha et al. 2011). Um caminho para lidar com tal complexidade tem sido a identificação de características mais propensas a participar dos processos ambientais, tais quais as que controlam a aquisição, uso e disponibilidade de recursos limitantes, as que interferem na estrutura das teias alimentares ou as que afetam a ocorrência e magnitude dos distúrbios (Tilman 2001).

Se os organismos podem ser taxonomicamente similares, é possível que haja indivíduos funcionalmente equivalentes ou redundantes (Naeem and Wright 2003). Características importantes em determinado processo ecológico podem ser compartilhadas entre muitas espécies, que, por sua vez, formariam grupos que utilizam um recurso ou que se comportam de modo parecido sob um gradiente de circunstâncias (Gitay and Noble 1997, Naeem and Wright 2003, Bruno and Olivier 2007). A identificação de tais atributos compartilhados pode conduzir à elucidação de padrões não evidenciados pelas mudanças na riqueza de espécies, o que teria amplo valor preditivo (Willians et al. 2010).

Desse modo, os ecólogos têm identificado populações-chave, histórias de vida e atributos ecológicos que influenciam as respostas das espécies às mudanças na paisagem (Willians et al. 2010). Há um longo histórico de tentativas de formulações de agrupamentos a partir de tais parâmetros, contudo, permanecem muitos obstáculos no desenvolvimento de métodos efetivos de classificação funcional (Gitay and Noble 1997, Petchey and Gaston 2002, Naeem and Wright 2003, Petchey and Gaston 2006, Petchey and Gaston 2007).

Como exemplo, a necessidade de se compreender melhor e predizer os efeitos mútuos entre os distúrbios ambientais, funcionamento do ecossistema e a biodiversidade, tem aumentado o interesse em estudos de assembleias de plantas sob o foco funcional (Bruno and Olivier 2007). De acordo com Júnior and Pillar (2004), desde 1993, no Global Change and 
Terrestrial Ecosystems (GCTE), principal núcleo de estudos do International Geosphere-Biosphere Programme (IGBP), têm sido organizados grupos que acreditam que a identificação de tipos funcionais de plantas é essencial para a construção de modelos preditivos globais sobre o impacto das alterações climáticas na vegetação.

Observa-se uma ampla conotação para o termo tipos funcionais de plantas (plant functional type - PFT). Pillar and Sosinski (2003), Santos et al. (2006) e Müller et al. (2007) adotam a definição de que os PFT correspondem a um grupo de plantas que, de modo não respectivo à sua filogenia, são similares em um conjunto de características e em suas associações a determinadas variáveis ambientais. Estudos têm demonstrado que características similares de plantas estão associadas com respostas específicas em diferentes fitofisionomias, o que se deve a um grau de convergência entre os atributos que condicionam o desempenho na comunidade (Louaut et al. 2005).

Desse modo, a identificação de tipos funcionais baseada em características de fácil reconhecimento, poderia compreender uma poderosa ferramenta para a compreensão das respostas das plantas (Louaut et al. 2005). Devido à alta relação entre estrutura e função, tem sido empregado o uso de atributos estruturais visíveis como substitutos aos caracteres funcionais, além disso, têm sido amplamente utilizadas características básicas ou a associação de atributos fisiológicos (Louaut et al. 2005, Müller et al. 2007). Como exemplo, o cerne central do estudo de Bruno and Olivier (2007) compreendeu a classificação das espécies de plantas por seus atributos morfológicos, fisiológicos e fenológicos, ao invés de adotarem uma abordagem restrita à filogenia do grupo.

Além disso, considerando-se os atributos dos organismos e sistemas, as características funcionais podem ser separadas em dois principais grupos (Naeem and Wright 2003, Violle et al. 2007, Brooks et al. 2012): a) atributos funcionais de efeitos, ou atributos de efeito, os quais se relacionam a uma dada função mensurada, como exemplo de características associadas ao ciclo do nitrogênio dentre as comunidades no solo, pelo volume total acumulado desse elemento químico; e b) atributos funcionais de resposta, ou atributos de resposta, que são mais importantes na determinação da estabilidade e resiliência da biota, e que correspondem à reação de um organismo a uma mudança ambiental, como características de tolerância à seca em ambientes áridos. Essa perspectiva é ampliada para os próprios grupos funcionais em Díaz and Cabido (2001), que apresentam os conceitos de tipos funcionais de efeito ou de resposta, grupos determinados pelas distintas e respectivas classes de atributos.

A compreensão da relação entre essas duas classes é valiosa por corresponder a um modo consistente de entendimento de mudanças na dinâmica ecossistêmica (Brooks et al. 2012). Contudo, é sabido que a escolha de um conjunto específico de atributos condiciona a perda de informações e a não visualização de todos os aspectos funcionais (Veríssimo et al. 2012). Dessa forma, uma seleção adequada é um passo fundamental, uma vez que o elevado número e diversidade de atributos podem acarretar em distintas representações de uma mesma assembleia (Tilman 2001, Veríssimo et al. 2012).

Tal seleção deve estar sujeita a um equilíbrio entre a eficácia de se descrever a variabilidade nas funções ecossistêmicas e o tempo e esforço necessários para o levantamento dos dados, atendendo aos seguintes critérios: a) os atributos devem apresentar importância funcional em observação à literatura; b) deve-se ter em consideração o julgamento de especialistas e conhecimento sobre a comunidade em estudo; e c) deve haver disponibilidade de informação sobre tais atributos (Veríssimo et al. 2012).

Devido às restrições mencionadas não há, ainda, um método único para a medida da diversidade funcional, contudo, o desenvolvimento de estratégias efetivas para a classificação, quantificação e manipulação da diversidade de base funcional é um importante passo para as pesquisas de relação entre a biodiversidade e o funcionamento do ecossistema (Tilman 2001, Petchey and Gaston 2002, Naeem and Wright 2003, Petchey and Gaston 2006, Petchey and Gaston 2007). Várias medidas estão aparecendo, as quais diferem na informação que contêm e na maneira com que quantificam a diversidade (Cianciaruso et al. 2009), tais medidas são usualmente representadas por índices baseados no conjunto de atributos dos organismos, havendo o consenso de que a diversidade funcional pode ser compreendida em diferentes componentes ligeiramente análogos à diversidade de espécies: riqueza, dispersão, similaridade e divergência funcionais (Manson et al. 2005, Gerisch et al. 2012, van der Linden et al. 2012).

Esses parâmetros são independentes entre si, 
mas também complementares na representação das propriedades de uma comunidade, devendo ser considerados simultaneamente (Gerisch et al. 2012). A observação da literatura indica que, com a exceção de poucos estudos que calculam tais medidas de diversidade funcional dentre as comunidades, muitos métodos de classificação são de natureza subjetiva, e poucos trabalhos distinguem atributos funcionais de efeitos e de respostas (Naeem and Wright 2003). Uma compreensão mais ampla sobre a evolução dos métodos de agrupamento funcional, meios de se calcular a diversidade funcional ou índices relacionados, assim como o que os mesmos mensuram, podem ser encontrados dentre os seguintes trabalhos: Lavorel et al. (1997), Pillar and DePatta (1999), Petchey and Gaston (2002), Pillar and Sosinsk (2003), Manson et al. (2005), Petchey and Gaston (2006), Petchey and Gaston (2007), Lavorel et al. (2008), Pillar et al. (2009), De Bello (2012), Dalerum et al. (2012), Gerisch et al. (2012), van der Linden et al. (2012).

Apesar de haver pouco consenso sobre as muitas abordagens utilizadas, revisões indicam considerável convergência, havendo três maiores métodos de classificação funcional (Gitay and Noble 1997, Gitay et al. 1999, Naeem and Wright 2003): a) subjetivo, no qual os grupos são livremente determinados por suas interações bióticas ou nichos; b) dedutivo, no qual, a priori, os autores deduzem os atributos assumidos por terem implicação direta no processo ecológico em estudo (Gitay and Noble 1997, Gitay et al. 1999, Müller et al. 2007); e c) definidos por dados, quando os grupos são determinados por modelos matemáticos ou métodos estatísticos que buscam segregações significativas dentre o conjunto de atributos (Gitay and Noble 1997, Gitay et al. 1999, Júnior and Pillar 2004, Müller et al. 2007).

A definição de grupos funcionais por dados pode ser realizada por uma ampla faixa de métodos de classificação, nos quais os grupos são usualmente mais precisos e preferíveis em relação aos demais métodos mencionados. Por assemelhar-se a um padrão taxonômico de agrupamento, esse método também pode possibilitar hierarquizações, a exemplo do uso de distâncias interespecíficas entre os atributos (Gitay and Noble 1997). Entretanto, como se têm acumulado índices de diversidade funcional em estudos de comunidade, devem-se evitar grandes correlações entre estas medidas e a diversidade taxonômica, por isso ser pouco útil no desenvolvimento de práticas complementares de predição das respostas da comunidade às mudanças ambientais (Gitay and Noble 1997, De Bello 2012).

Tal interferência indica uma necessidade de estudos sobre a relação entre esses dois componentes, como apontado por MacArthur em 1950 (Barbault 1995, Naeem and Wright 2003). Como exemplo, em um estudo sobre besouros do solo amostrados em campos inundáveis ao longo do rio Elbe, na Alemanha, Gerish et al. (2012) encontraram correlação entre a riqueza de espécies e a diversidade funcional dependente do índice de diversidade funcional utilizado. Contudo, em um estudo baseado em uma comunidade construída por simulação, a partir de dados empíricos, Lyashevska and Farnswhorth (2012) não encontraram relações entre as métricas de medidas para as diversidades funcional, taxonômica e riqueza de espécies, de modo que possíveis correlações entre esses índices podem refletir a influência de fatores ambientais não abrangidos na simulação.

\section{COMPARAÇÕES ENTRE AS PERSPECTIVAS TAXONÔMICA E FUNCIONAL}

Segundo Lyashevska and Farnswhorth (2012), a biodiversidade seria essencialmente tridimensional, com seus eixos correspondendo às diversidades funcional (suportada pela função ecológica), taxonômica (representante genético) e estrutural (baseada na abundância das espécies). Essa perspectiva é mais abrangente do que a visão usual da biodiversidade como um simples indicador de mudanças ecológicas, para a sua contemplação como uma informação requerida para uma descrição abrangente ou para representação da comunidade como um sistema vivo e complexo.

Desse modo, os componentes bióticos relacionados à magnitude e à variabilidade das alterações na biodiversidade podem ser distribuídos entre quatro fatores principais: a) a composição; b) a abundância; c) as características funcionais; e d) as interações bióticas entre as espécies (Naeem and Wright 2003). Contanto, grande parte da pesquisa em biodiversidade se concentra na diversidade taxonômica, representada pelo levantamento de listas de riqueza e abundância relativa de espécies, pelos estudos de distância filogenética cumulativa e por padrões espaciais e temporais da distribuição (Díaz and Cabido 2001, Andresen 2003, Naeem and Wright 
2003). Pouco se sabe sobre como o número de espécies presentes em uma comunidade se relaciona às funções do ecossistema (Gerisch et al. 2012). Como exemplo, estudos sobre a fragmentação de ambientes florestais têm enfocado nos efeitos diretos de primeira ordem, como a indução de mudanças na abundância e diversidade, não abrangendo as consequências funcionais dessa alteração (Andresen 2003).

Apesar de representarem a relação entre a flutuação na abundância de espécimes e as mudanças no ecossistema, bases exclusivamente taxonômicas possuem carência na demonstração da estrutura natural da comunidade (Larsen et al. 2005, SilvaPereira and Santos 2006). A habilidade de tais métricas em refletir os efeitos antrópicos não está clara e sua aplicação condiciona uma série de preocupações, incluindo: a) dificuldades de classificação dos indivíduos ao nível de espécies; b) comparações inadequadas entre locais distintos; c) relações incoerentes aos distúrbios, uma vez que a relação entre diversidade e distúrbio permanece controversa; d) inabilidade de discernir entre distúrbios naturais ou antrópicos, e e) pouca informação fornecida sobre o funcionamento do ecossistema (Veríssimo et al. 2012).

Tal lacuna decorre do fato de assumir-se um peso igual para todas as espécies na comunidade, independentemente de suas características, requerimentos e/ou funções (Podigaiski et al. 2011, Gerisch et al. 2012). Os métodos tradicionais de medida de biodiversidade não são capazes de refletir plenamente as relações entre as flutuações ambientais, a ocorrência de espécies e os processos na comunidade, uma vez que tal dinamismo não é definido apenas pelo componente filogenético de sua biota, mas também por seus atributos funcionais (Naeem and Wright 2003, Gerisch et al. 2012). Desse modo, devem-se incluir métricas que contemplem diretamente os componentes funcionais, considerando suas propriedades estruturais, sua organização e a relação que apresentam com os processos e serviços ecossistêmicos (Podigaiski et al. 2011).

Estudos determinados apenas sobre uma única espécie, ou grupos de espécies, podem não evidenciar as mudanças drásticas na composição da comunidade ou nos processos ecológicos (Andresen 2003), de modo que importantes aspectos funcionais, afetados independentemente ou em adição à riqueza de espécies, não têm sido observados, podendo levar a conclusões errôneas (Naeem and Wright 2003). Além disso, do ponto de vista ecológico, a biodiversidade ultrapassa os conceitos de diversidade e riqueza de espécies, sendo dada ênfase ao nível de sistemas (metapopulações, comunidade, ecossistema, paisagem), com o componente funcional compreendendo um importante vetor de esclarecimentos (Barbault 1995, Veríssimo et al. 2012).

Dentre outras abordagens, a riqueza de espécies pode estar relacionada à diversidade funcional pelo efeito da complementaridade de nichos, que perdiz que com uma maior diversidade condiciona uma maior faixa de características funcionais presentes, suportando mais oportunidades de uso eficiente de recursos em um ambiente com variações temporais e espaciais (Díaz and Cabido 2001). Contudo, tal relação parece não ser linear e a complexidade dos efeitos combinados da riqueza e funcionalidade é de difícil representação empírica (Cardinale et al. 2000, Díaz and Cabido 2001, Dalerum et al. 2012), de modo que dados observacionais que suportam tal relação são frequentemente equívocos em sua representação (Cardinale et al. 2000, Schwartz et al. 2000).

A diversidade biológica pode ser mais bem representada pelas particularidades interespecíficas na eficiência e uso de recursos, que resultam em distinções na distribuição de espécies (Barbault 1995). Um táxon (ou um conjunto de poucos taxa) pode apresentar uma influência desproporcional nos processos (Cardinale et al. 2000), sendo mais importante do que qualquer medida cumulativa de diversidade (Flynn et al. 2011). Tal relação ocorre, por exemplo, em espécies de competidores dominantes, espécies-chave ou com características biológicas exclusivas, as quais não podem ser diferenciadas pelo agrupamento taxonômico tradicional (Cardinale et al. 2000).

Observa-se que a diversidade funcional parece ser mais relevante em muitos estudos em que ela é examinada simultaneamente à diversidade taxonômica (Naeem and Wright 2003), havendo um grande consenso de que a diversidade funcional pode determinar amplamente o funcionamento do ecossistema (Díaz and Cabido 2001). Desse modo, as pesquisas que possuem foco exclusivo no número de espécies, ou nas espécies por si, não compreendem o melhor caminho para se esclarecer questões referentes à biodiversidade, sendo mais pertinente explanar-se sobre os motivos da ocorrência, variação ou persistência da biodiversidade local (Barbault 1995, Purvis and Hector 2000). 
Complementarmente, há dois argumentos principais para que as pesquisas centradas no caráter específico possuam uma associação às análises funcionais (Cardinale et al. 2000, Bruno and Olivier 2007): a) as classificações de habitat centradas em espécies não podem ser prontamente utilizadas para outras regiões que possuam um conjunto de espécies distinto, por outro lado, uma classificação funcional baseada nos atributos de história de vida possibilitam a comparação de resultados entre continentes; e b) um objetivo comum para as práticas de manejo é promover condutas de conservação, como grupos funcionais são compostos por espécies que apresentam atributos biológicos similares, espera-se que a resposta ao manejo também seja similar entre as espécies (Bruno and Olivier 2007).

Estudos têm considerado os efeitos do número de características funcionais ou da redundância funcional, e mais recentemente, os efeitos da riqueza e composição de espécies na resiliência da diversidade funcional. Entretanto, ainda não têm sido analisados simultaneamente os efeitos combinados da riqueza de espécies, do número de atributos funcionais e de sua diversidade dentre as espécies (Dalerum et al. 2012). Além disso, estudos recentes têm sugerido que a diversidade filogenética (a história evolucionária particular de uma comunidade) pode ser utilizada como um referencial da diversidade funcional, uma vez que a diversificação evolutiva pode acarretar em diversificação de atributos e maior complementaridade de nichos (Flynn et al. 2011, Trindade-Filho et al. 2012).

Desse modo, métodos dinâmicos e integrativos de pesquisa podem parecer mais eficientes por possibilitarem a percepção das interações geosferabiosfera por meio dos mecanismos biológicos básicos, a exemplo de estudos de respostas funcionais primordiais, como a polinização e dispersão de sementes (Barbault 1995). Tornam-se necessários métodos de agrupamentos que representem os meios em que organismos influenciam a estrutura da comunidade e os elementos com os quais eles interagem (Purvis and Hector 2000, Andresen 2003).

Em uma pesquisa bibliográfica sobre a relação entre a riqueza de espécies e o funcionamento do ecossistema, realizada pelo Current Contents (Institute for Scientific Information Inc.), Biosis (Biological Abstracts Inc.) e Bio85 (Biological Abstracts Inc.), encontrou-se que o conjunto de citações combinando as expressões "biodiversity" e "ecosystem function" foi nulo de 1985 a 1990, apresentaram 18 citações entre os 1991 e 1994; e 76 entre 1995 e 1998 (Schwartz et al. 2000). Apesar do número crescente de trabalhos, permanece o debate sobre a natureza e motivos das relações entre a diversidade de espécies e as taxas de processos ecológicos, o qual tem resultado em poucas generalizações (Cardinale et al. 2000). Tais aproximações podem estar sendo limitadas por pouco se considerar as influências de processos biogeográficos sobre a comunidade, que também participam do controle da composição, distribuição e abundância de espécies (Naeem and Wright 2003).

Havendo poucos esforços para se determinar em quais situações a diversidade de espécies pode importar no funcionamento do ecossistema, os ecólogos têm feito inferências, permanecendo crucial a necessidade das descrições e documentações taxonômicas como bases para as pesquisas (Barbault 1995, Cardinale et al. 2000). Além disso, pode não haver uma relação única e generalizável entre a diversidade de espécies e as taxas dos processos ecológicos, uma vez que em muitos contextos, a dicotomia entre diversidade ou composição de espécies pode ser errônea (Cardinale et al. 2000, Bruno and Olivier 2007).

\section{ASSOCIAÇÃO DE DIFERENTES PERSPECTIVAS}

É possível que a melhor contribuição obtida pelas abordagens de atributos funcionais de plantas em estudos de ecossistemas e comunidades tenha sido um conhecimento maior sobre a estrutura da comunidade e o funcionamento do ecossistema. Contudo, a miscigenação entre as perspectivas baseadas em espécies e em tipos funcionais, tem o potencial de contribuir para o estudo de técnicas de manejo para a conservação da diversidade e dos serviços ecossistêmicos (Díaz and Cabido 2001). Portanto, para o esclarecimento das relações entre o componente biótico e os mecanismos do ecossistema, parece ser mais promissora a adoção de estudos de grupos funcionais em perspectiva associativa, ao invés de substitutiva às classificações taxonômicas ou a parâmetros tradicionais da ecologia.

Tais estudos envolvem tipicamente o falseamento de hipóteses nulas sobre a independência entre as funções-chave e o número de espécies presentes na comunidade, sendo um importante objetivo 
a busca por aspectos e métodos quantitativos para caracterizar a relevância das espécies, tornando-se valiosa a identificação de espécies-chaves e de espécies redundantes (Duarte 2000, Jordán 2002).

Espécies redundantes compreendem organismos análogos em suas funções, tanto que o desaparecimento de algumas dessas não ocasiona perdas funcionais mensuráveis na comunidade (Duarte 2000). Por outro lado, espécies ou guildas-chave correspondem aos mais significativos componentes biológicos em determinado ambiente, cuja presença ou ausência pode alterar drasticamente a estrutura e dinâmica dos sistemas ecológicos (Brown and Heske 1990, Díaz and Cabido 2001, Jordán 2002).

Como exemplo, os animais vertebrados correspondem a apenas uma pequena fração da biomassa e do fluxo energético em muitos ambientes, mas através das relações de competição, mutualismo ou predação, eles podem promover grandes alterações nos processos bioquímicos, na composição de espécies e na estrutura do hábitat (Brown and Heske 1990). Tal relação suportou o estudo de Dalerum et al. (2013), no qual análises da diversidade funcional e filogénica em assembleia de carnívoros de grande porte foram utilizadas para dar suporte a estratégias de conservação desse grupo altamente propenso a extinção.

Desse modo, uma vez que os efeitos iniciais e mais previsíveis da atividade humana são direcionados à estrutura trófica, o estudo das teias alimentares e dos níveis tróficos parece promissor (Barbault 1995, Schwartz et al. 2000, Duff 2003). Além disso, a presença de espécies-chaves pode não evidenciar padrões ao nível de comunidade, devendo-se adotar medidas que abranjam simultaneamente os padrões emergentes na comunidade e a dinâmica interespecífica (Berlow et al. 2004). Aspectos considerados em estudos de modelagem da dinâmica populacional, através da identificação dos elementos relacionados às redes de interações entre espécies e à hierarquização de sistemas biológicos (Barbault 1995).

Nesse caso, o foco é direcionado às propriedades dinâmicas, ao invés de características estáticas, de modo que fortes elos podem ser demonstrados experimentalmente (Barbault 1995, Briand and Cohen 1987). Como apresentado por Brooks et al. (2012), os quais encontraram que as características de resposta em um nível trófico podem mediar efeitos em outro nível relacionado, ao testarem a habilidade de ervas cultivadas em responder de modo particular às perturbações de cultivo em acordo com suas características, e de afetarem diferencialmente o nível trófico superior de besouros Carabidae.

Um desafio compreende o registro de como a diversidade, seja ao nível específico ou funcional, varia entre os diferentes níveis tróficos, uma vez que a intensidade das alterações ambientais depende também do número de níveis presentes (Duff 2003). Por outro lado, o conhecimento associativo entre a estrutura das redes de interação e suas forças, pode possibilitar a identificação de processos-chave em outras porções da comunidade. Estudos baseados em atributos como taxas de forrageio, tamanho do corpo, taxa metabólica e de distribuição de biomassa, podem auxiliar amplamente na compreensão da relação entre o escopo teórico e o experimental, por explorarem caminhos de desenvolvimento de modelos e coeficientes biológicos a partir de dados empíricos de fácil obtenção (Berlow et al. 2004).

Contudo, a compilação de dados referentes aos atributos permanece como um desafio, sendo evidente a necessidade de um banco de dados central (Flynn et al. 2012). Como mencionado anteriormente, as maiores restrições ao conduzir análises baseadas em atributos são o tempo necessário para reunir informações relevantes, sua disponibilidade e a possibilidade de contradição dentre as diferentes fontes (Veríssimo et al. 2012).

Em uma revisão sobre os trabalhos publicados examinando a relação entre a diversidade funcional e o funcionamento do ecossistema, Naeem and Wright (2003) encontraram que quase a metade dos trabalhos, 44, contém um componente experimental, enquanto menos do que $10 \%$ dos estudos foram observacionais. Variados métodos têm sido utilizados para a classificação das espécies de acordo com seus atributos funcionais, com mais da metade destes estudos agrupando os organismos por sua forma de vida ou posição trófica.

Além disso, o elo entre a estrutura do ambiente e a biodiversidade de animais consumidores tem recebido pouca atenção (Liow et al. 2001, Duff 2003). Muitos grupos taxonômicos e tipos de habitat têm sido pouco representados, tornando difícil a formação de métodos gerais (Gerisch et al. 2012). Observa-se que a literatura ecológica referente a procedimentos de medidas e análises de atributos funcionais têm sido quase que exclusivamente desenvolvida para plantas em diferentes ecossistemas, usualmente terrestres (Walker et al. 1999, Díaz and Cabido 2001, Naeem 
and Wright 2003, Cianciaruso et al. 2009 e Vandewalle et al. 2010). Tal tendência dificulta a aplicabilidade em sistemas mais complexos ou multitróficos (Naeem and Wright 2003 e Walker et al. 1999).

A insuficiência de dados experimentais conduz à constatação de que o número de consumidores que desempenham um papel funcional importante nas comunidades é inferior ao de produtores (Liow et al. 2001, Duff 2003). Contudo, algum avanço tem sido feito, como o uso de índices de atributos funcionais em grupos animais (Villeger et al. 2013), ou a determinação de atributos para invertebrados (Colzani et al. 2013, Martínez et al. 2013, Petrin et al. 2013), correspondentes à morfologia, fisiologia, comportamento alimentar, uso e especialização do hábitat, estratégias de fuga de predadores ou de condições ambientais severas (Podgaiski et al. 2011, Hedde et al. 2012). Tais critérios têm sido comprovados como bons preditores das respostas às mudanças ambientais para comunidades de aves, insetos e zooplâncton (Gallego et al. 2012).

As plantas corresponderem a cerca de um sexto de todas as espécies descritas e os insetos correspondem a aproximadamente dois terços das mesmas, podendo estes, junto aos demais invertebrados, corresponder aos organismos mais importantes para a manutenção de processos vitais nos ecossistemas tropicais devido à sua ampla abundância e diversidade (Liow et al. 2001, Duff 2003, Warse 2006, Podgaiski et al. 2011). As interações entre plantas e invertebrados são componentes predominantes do ecossistema terrestre, e dentre elas, a polinização relaciona cerca de duzentas e cinquenta mil espécies de angiospermas a um número similar de insetos (Warse 2006).

Os insetos que visitam flores, os quais são principalmente representados pelos Hymenoptera Apoidea, estão possivelmente relacionados à evolução e diversificação das angiospermas (Grimaldi 1999, Grimaldi and Engel 2005, Chacoff and Aizen 2006, Silva-Pereira and Santos 2006). As pesquisas das diversidades conjuntas de consumidores e plantas podem ser amplamente informativas e permanecem como uma importante via (Hochberg and Hawkins 1992, Duff 2003). Como exemplo, para elucidar questões referentes às respostas das abelhas (Apidae) à perda do habitat natural, os estudos deveriam incluir com maior profundidade os atributos da história natural dos indivíduos em uma perspectiva funcional (Cane 2001, Larsen et al. 2005 e Ewers and Didham 2006).
Ainda dentre os invertebrados terrestres, as formigas representam um táxon ideal para se testar padrões de organização das comunidades a partir de atributos (Neves et al. 2013). Esses organismos usualmente apresentam hábitos generalistas, havendo muitas evidências de que as comunidades são largamente estruturadas pela competição (Podgaiski et al. 2011). Por sua vez, os insetos parasitóides compreendem cerca de $20 \%$ de todas as espécies de sua classe, em conjunto aos predadores invertebrados, são dominantes em redes tróficas e possuem maior diversidade específica do que os vertebrados (Hochberg and Hawkins1992). Contudo, sua importância funcional para a comunidade é pouco compreendida (Duff 2003), de modo que tal questão representa um grande passo no conhecimento da dinâmica da diversidade das comunidades terrestres (Hochberg and Hawkins1992).

Geralmente negligenciadas ao nível de comunidades, as implicações de doenças infecciosas e interações de parasitismo e predação compreendem outro campo de pesquisas promissor. Pequenas mudanças, como no clima local, podem intensificar a extinção de algumas espécies e promover a predominância de outras, pelo benefício fisiológico ou pelo declínio de populações de competidores, predadores ou parasitas. Tais relações podem ter um impacto considerável na dinâmica da população de hospedeiros e, mais amplamente, na composição de espécies e estrutura da comunidade de plantas e animais (Barbault 1995).

\section{CONCLUSÕES}

O intuito de compreender as implicações das mudanças na biodiversidade sobre o ecossistema tem demonstrado que variações nas diversidades taxonômica e funcional podem acarretar alterações nos mecanismos ambientais, uma vez que a variedade de diferentes processos ecológicos é influenciada pelo número e tipos de espécies viventes em determinado local (Tilman 2001). Desse modo, o entendimento dos padrões de mudanças na biodiversidade e suas consequências funcionais pode aumentar nossa habilidade para predizer respostas, além de proteger os processos ambientais (Brown and Heske 1990, Larsen et al. 2005).

Como condutas aparentemente divergentes podem compreender, na realidade, parcelas complementares, a clareza quanto às hipóteses 
levantadas e quanto à abrangência dos conceitos adotados facilita a comunicação entre diferentes programas de pesquisa, possibilitando medidas sintéticas (Naeem and Wright 2003, Berlow et al. 2004). Uma vez que um ponto de vista pode não ser capaz de abranger todas as respostas, é mais interessante avaliar quais questões relacionadas a determinado tema são relevantes, para que cada aspecto seja avaliado adequadamente, antes que se recomende a aplicação de determinado preceito em detrimento do outro (Cameron 2002, Berlow et al. 2004).

Um caminho plausível corresponde à síntese de pesquisas centradas em taxonomia, biogeografia, e em ecologia funcional de comunidades e de ecossistemas, uma vez que muitas dessas áreas de conhecimento estão relativamente bem desenvolvidas (Naeem and Wright 2003). Tais medidas contribuem para evitaremse falsos debates, proporcionando a conversão de variáveis em resultados, além da associação dos conteúdos teórico e empírico, conduzindo a uma ciência eficaz e preditiva (Naeem and Wright 2003, Berlow et al. 2004, Dalerum et al. 2012).

Como não há uma maneira única de se mensurar a diversidade funcional, um importante desafio compreende o estabelecimento e adoção de uma terminologia concisa, de protocolos de pesquisa aceitáveis que possibilitem a generalização de tais práticas aos mais distintos níveis de organização dos seres vivos (Tilman 2001). Contudo, a presente revisão demonstra que a Ecologia Funcional se fundamenta em diversos conceitos recentes que têm sido utilizados para diferentes fins, apresentando distintas facetas. Apesar de parte desses conceitos já possuir significado mais universal, muitos se encontram em construção. Desse modo, a adoção correta de determinado conceito é amplamente suportada pelo conhecimento sobre as diferentes perspectivas em que determinado termo pode ser adotado de forma coerente, o que se reflete no histórico de seu desenvolvimento, sendo de grande importância expressar-se de modo claro e fundamentado para evitarem-se interpretações errôneas ou o surgimento de termos redundantes.

AGRADECIMENTOS: Agradeço aos amigos e pesquisadores que me auxiliaram com sugestões para a redação e submissão desse trabalho e à agência de fomento CAPES pela bolsa concedida durante a realização de meu doutorado.

\section{REFERÊNCIAS}

Andresen, E. 2003. Effect of forest fragmentation on dung beetle communities and functional consequences for plant regeneration. Ecography 26:87-97. http://dx.doi.org/10.1034/ j.1600-0587.2003.03362.x

Barbault, R. 1995. Biodiversity dynamics: from population and community ecology approaches to a landscape ecology point of view. Landscape and Urban Planning 31:89-98. http://dx.doi.org/10.1016/0169-2046(94)01038-A

Berlow, E. L., A.-M. Neutel, J. E. Cohen, P. C. De Ruiter, B. Ebenman, M. Emmerson, J. W. Fox, V. A. A. Jansen, J. I. Jones, G. D. Kokkoris, D. O. Logofet, A. J. McKane, J. M. Montoya, and O. Petchey. 2004. Interaction strengths in food webs: issues and opportunities. Journal of Animal Ecology 73:585598. http://dx.doi.org/10.1111/j.0021-8790.2004.00833.x

Briand, F., and J. E. Cohen. 1987. Environmental Correlates of Food Chain Length. Science 238:956-960. http:// dx.doi.org/10.1126/science.3672136

Brown, J. H., and E. J. Heske, E. J. 1990. Control of a Desert-Grassland Transition by a Keystone Rodent Guild. Science 250:1705-1707. http://dx.doi.org/10.1126/science.250.4988.1705

Brooks, D. R., J. Storkey, S. J. Clark, L. G. Firbank, S. Petit, and I. P. Woiwod. 2012. Trophic links between functional groups of arable plants and beetles are stable at a national scale. Journal of Animal Ecology 81:4-13. http://dx.doi.org/10.1111/ j.1365-2656.2011.01897.x

Bruno, H., and H. Olivier. 2007. Using life-history traits to achieve a functional classification of habitats. Applied Vegetation Science 10:73-80. http://dx.doi.org/10.1658/14022001(2007)10[73:ULTTAA]2.0.CO;2

Cameron, T. 2002. 2002: The year of the 'diversityecosystem function' debate. Trends in Ecology and Evolution 17:495-496. http://dx.doi.org/10.1016/S0169-5347(02)02618-6

Cane, J. H. 2001. Habitat fragmentation and native bees: a premature verdict? Conservation Ecology 5(1):3. <http:// www.consecol.org/vol5/iss1/art3/>. (Acesso em 12/03/2012).

Cardinale, B. J., K. Nelson, and M. A. Palmer. 2000. Linking species diversity to the functioning of ecosystems: on the importance of environmental context. Oikos 91:175-183. http:/ /dx.doi.org/10.1034/j.1600-0706.2000.910117.x

Chacoff, N. P., and M. A. Aizen. 2006. Edge effects on flower-visiting insects in grapefruit plantations bordering premontane subtropical forest. Journal of Applied Ecology 43:1827. http://dx.doi.org/10.1111/j.1365-2664.2005.01116.x

Cianciaruso, M. V., I. A. Silva,. and M. A. Batalha. 2009. Diversidades filogenética e funcional: novas abordagens para a Ecologia de comunidades. Biota Neotropica 9:93-103. http:// dx.doi.org/http://www.biotaneotropica.org.br/v9n3/pt/ abstract?article+bn01309032009

Clements, F. E. 1916. Plant succession: An analysis of the development of vegetation. Washington, DC: Carnegie Institute. Washington Publ. 242:3-4.

Colzani, E., T. Siqueira, M. T. Suriano, and F. O. Roque. 2013. Responses of Aquatic Insect Functional Diversity to Landscape Changes in Atlantic Forest. Biotropica 45:343-350. http://dx.doi.org/10.1111/btp.12022

Dalerum, F. 2013. Phylogenetic and functional diversity in large carnivore assemblages. Proceedings of the Royal Society B 280:1-9. http://dx.doi.org/http://dx.doi.org/10.1098/ rspb.2013.0049

Dalerum, F., E. Z. Cameron, K. Kunkel, and M. J. Somers. 
2012. Interactive effects of species richness and species traits on functional diversity and redundancy. Theoretical Ecology 5:129139. http://dx.doi.org/10.1007/s12080-010-0104-y

De Bello, F. 2012. The quest for trait convergence and divergence in community assembly: are null-models the magic wand? Global Ecology and Biogeography 21:312-317. http:// dx.doi.org/10.1111/j.1466-8238.2011.00682.x

De Bello, F., S. Lavorel, S. Díaz, R. Harrington, J. H. C. Cornelissen, R. D. Bardgett, M. P. Berg, P. Cipriotti, C. K. Feld, D. Hering, P. M. Silva, S. G. Potts, L. Sandin, J. P. Sousa, J. Storkey, D. A. Wardle, and P. A. Harrison. 2010. Towards an assessment of multiple ecosystem processes and services via functional traits. Biodiversity and Conservation 19:2873-2893. http://dx.doi.org/10.1007/s10531-010-9850-9

Díaz, S., and M. CABIDO. 2001. Vive la différence: plant functional diversity matters to ecosystem processes. Trends in Ecology and Evolution 16:646-655. http://dx.doi.org/10.1016/ S0169-5347(01)02283-2

Duarte, C. M. 2000. Marine biodiversity and ecosystem services: an elusive link. Journal of Experimental Marine Biology and Ecology 250:117-131. http://dx.doi.org/10.1016/S00220981(00)00194-5

Duff, J. E. 2003. Biodiversity loss, trophic skew and ecosystem functioning. Ecology Letters 6:680-687. http:// dx.doi.org/10.1046/j.1461-0248.2003.00494.x

Ewers, R. M., and R. K. Didham. 2006. Confounding factors in the detection of species responses to habitat fragmentation. Biological Reviews 81:1-26. http://dx.doi.org/ 10.1017/S1464793105006949

Fenster, C. B., W. S. Armbruster, P. Wilson, M. R. Dudash, and J. D. Thomson. 2004. Pollinization syndromes and flora specialization. Annual Review of Ecology, Evolution, and Systematics 35:375-403. http://dx.doi.org/10.1146/ annurev.ecolsys.34.011802.132347

Flynn, D. F. B., N. Mirotchnick, M. Jain, M. I. Palmer, and S. Naeem. 2011. Functional and phylogenetic diversity as predictors of biodiversity - ecosystem-function relationships. Ecology 92:1573-1581. http://dx.doi.org/http://dx.doi.org/10.1890/ $10-1245.1$

Gallego, I., T. A. Davidson, E. Jeppesen, C. PérezMartínez, P. Sánchez-Castillo, M. Juana, F. Fuentes-Rodriguez, D. León, P. Peñalverg, J. Toja, and J. J. Casas. 2012. Taxonomic or ecological approaches? Searching for phytoplankton surrogates in the determination of richness and assemblage composition in ponds. Ecological Indicators 18:575-585. http://dx.doi.org/ 10.1016/j.ecolind.2012.01.002, doi:10.1016/j.ecolind.2012.01.002

Gerisch, M., V. Agostinelli, K. Henle, and F. Dziock. 2012. More species, but all do the same: contrasting effects of flood disturbance on ground beetle functional and species diversity. Oikos 121:508-515. http://dx.doi.org/10.1111/j.16000706.2011.19749.x

Gitay, H., and I. R. Noble. 1997. What are functional types and how should we seek them? Pages 3-19 in: T. M. Smith, H. H. Shugart, and F. I. Woodward, editors. Plant Functional Types - Their relevance to ecosystem properties and global changes. Cambridge University Press. Cambridge, USA.

Gitay, H., I. R. Noble, and J. H. Connell. 1999. Deriving functional types for rain-forest trees. Journal of Vegetation Science 10:641-650. http://dx.doi.org/10.2307/3237079

Gleason, H. A. 1939. The individualistic concept of the plant association. American Midland Naturalist 21:92-110. http:/ /dx.doi.org/10.1177/030913339602000205
Grimaldi, D. 1999. The Co-Radiations of Pollinating Insects and Angiosperms in the Cretaceous. Annals of the Missouri Botanical Garden 86:373-406. http://dx.doi.org/10.2307/2666181

Grimaldi, D., and M. S. Engel. 2005. Diverity and Evolution. Pages 1-41 in: Grimaldi, D., and M. S. Engel, editors. Evolution of the Insects. Cambridge University Press, New York, USA.

Grönroos, M., and J. Heino. 2012. Species richness at the guild level: effects of species pool and local environmental conditions on stream macroinvertebrate communities. Journal of Animal Ecology 81:679-691. http://dx.doi.org/10.1111/j.13652656.2011.01938.x

Hedde, M., F. Van Oort, and I. Lamy. 2012. Functional traits of soil invertebrates as indicators for exposure to soil disturbance. Environmental Pollution 164:59-65. http://dx.doi.org/ 10.1016/j.envpol.2012.01.017

Hochberg, M. E., and B. A. Hawkins. 1992. Refuges as a predictor of parasitoid diversity. Science 255:973-976. http:// dx.doi.org/10.1126/science.255.5047.973

Jordan, F. 2002. Searching for keystones in ecological networks. Oikos 99:607-612. http://dx.doi.org/10.1126/ science. 255.5047 .973

Júnior, E. E. S., and V. D. Pillar. 2004. Respostas de tipos funcionais de plantas à intensidade de pastejo em vegetação campestre. Pesquisa Agropecuária Brasileira 39:1-9. http:// dx.doi.org/http://dx.doi.org/10.1590/S0100-204X2004000100001

Larsen, T. H., M. N. Williams, and C. Kremen. 2005. Extinction order and altered community structure rapidly disrupt ecosystem functioning. Ecology Letters 8:538-547. http:// dx.doi.org/10.1111/j.1461-0248.2005.00749.x

Lavorel, S., K. Grigulis, S. McIntyre, N. S. G. Williams, D. Garden, J. Dorrough, S. Berman, F. Quétier, A, Thébault, and A. Bonis. 2008. Functional Ecology 22:134-147. http://dx.doi.org/ 0.1111/j.1365-2435.2007.01339.x

Lavorel, S., S. McIntyre, and T. D. A. Forbes. 1997. Plant, functional classifications: from general groups to specific groups based on response to disturbance. TREE 12:474-478. http:// dx.doi.org/http://dx.doi.org/10.1016/S0169-5347(97)01219-6

Liow, L. H., N. S. Sodhi, and T. Elmqvist, 2001. Bee diversity along a disturbance gradient in tropical lowland forests of south-east Asia. Journal of Applied Ecology 38:180-192. http:/ /dx.doi.org/10.1046/j.1365-2664.2001.00582.x

Louaut, F., V. D. Pillar, J. Aufrère, E. Garnier, and J.-F. Soussana. 2005. Plant traits and functional types in response to reduced disturbance in a semi-natural grassland. Journal of Vegetation Science 16:151-160. http://dx.doi.org/10.1111/j.16541103.2005.tb02350.x

Lyashevska, O., and K. D. Farnsworth. 2012. How many dimensions of biodiversity do we need? Ecological Indicators 18:485-492. http://dx.doi.org/10.1016/j.ecolind.2011.12.016

Mason, N. W. H., D. Mouillot, W. G. Lee, and J.B. Wilson. 2005. Functional richness, functional evenness and functional divergence: the primary components of functional diversity. Oikos 111:112-118. http://dx.doi.org/10.1111/j.0030-1299.2005.13886.x

Martínez, A., A. Larrañaga, A. Basaguren, J. Pérez, C. Mendoza-Lera, and J. Pozo. 2013. Stream regulation by small dams affects benthic macroinvertebrate communities: from structural changes to functional implications. Hydrobiologia 711:31-42. http://dx.doi.org/10.1007/s10750-013-1459-z

Müller, S. C., G. E. Overbeck, J. Pfadenhauer, and V. D. Pillar. 2007. Plant functional types of woody species related to fire disturbance in forest-grassland ecotones. Plant Ecology 189:114. http://dx.doi.org/10.1007/s11258-006-9162-z 
Naeem, S., and J. P. Wright. 2003. Disentangling biodiversity effects on ecosystem functioning: deriving solutions to a seemingly insurmountable problem. Ecology Letters 6:567579. http://dx.doi.org/10.1046/j.1461-0248.2003.00471.x

Neves, F. S., K. S. Queiroz-Dantas, W. D. Rocha, and J. H. C. Delabie. 2013. Ants of three adjacent habitats of a transition region between the cerrado and caatinga biomes: the effects of heterogeneity and variation in canopy cover. Neotropical Entomology 42:258-268. http://dx.doi.org/10.1007/s13744-013-0123-7

Petchey, O. L., and K. J. Gaston. 2002. Functional diversity (FD), species richness and community composition. Ecology Letters 5:402-411. http://dx.doi.org/10.1046/j.14610248.2002.00339.x

Petchey, O. L., and K. J. Gaston. 2006. Functional diversity: back to basics and looking forward. Ecology Letters 9:741-758. http://dx.doi.org/10.1111/j.1461-0248.2006.00924

Petchey, O. L., and K. J. Gaston. 2007. Dendrograms and measuring functional diversity. Oikos 116:1422-1426. http:// dx.doi.org/10.1111/j.2007.0030-1299.15894.x

Petrin, Z., J. E. Brittain, and S. J. Saltveit. 2013. Mayfly and stonefly species traits and species composition reflect hydrological regulation: a meta-analysis. The Society for Freshwater Science 32:425-437. http://dx.doi.org/http://dx.doi.org/ 10.1899/11-172.1

Pillar, V. D. 1999. On the identification of optimal plant functional types. Journal of Vegetation Science 10:631-640. http:/ /dx.doi.org/10.2307/3237078

Pillar, V. D., and E. E. Sosinski. 2003. An improved method for searching plant functional types by numerical analysis. Journal of Vegetation Science 14:323-332. http://dx.doi.org/10.1111/ j.1654-1103.2003.tb02158.x

Pillar, V. D., L. S. Duarte, E. E. Sosinski, and F. Joner. 2009. Discriminating trait-convergence and trait-divergence assembly patterns in ecological community gradients. Journal of Vegetation Science 20:334-348. http://dx.doi.org/10.1111/j.16541103.2009.05666.x

Podgaiski, L. R., M. S. Mendonça Jr., and Pillar, V. D. 2011. O uso de atributos funcionais de invertebrados terrestres na ecologia: o que, como e por quê? Oecologia Australis 15:835-853.

Purvis, A., and A. Hector. 2000. Getting the measure of biodiversity. Nature 405:212-219. http://dx.doi.org/10.1038/ 35012221

Rocha, M. R., U. Gaedke, and D. A. Vasseur. 2011. Functionally similar species have similar dynamics. Journal of Ecology 9:1453-1459. http://dx.doi.org/10.1111/j.13652745.2011.01893.x

Santos, B. R. C., M. A. Silva, R. B. Medeiros, C. Blanco, E. Sosinski, V. D. Pillar, J. C. Saibro, and R. S. Rodrigues. 2006. Interação comportamento de pastejo x dinâmica de tipos funcionais em pastagem natural na Depressão Central do Rio Grande do Sul. Revista Brasileira de Zootecnia 35:1897-1906. http://dx.doi.org/10.1590/S1516-35982006000700004

Schwartz, M. W., C. A. Brigham, J. D. Hoeksema, K. G. Lyons, M. H. Mills,. and P. J. Van Mantgem. 2000. Linking biodiversity to ecosystem function: implications for conservation ecology. Oecologia 122:297-305. http://dx.doi.org/10.1007/ s004420050035

Semenova, G. V., and E. Van der Maarel. 2000. Plant functional types - a strategic perspective. Journal of Vegetation Science 11:917-922. http://dx.doi.org/10.2307/3236562

Silva-Pereira, V., and G. M. M. Santos. 2006. Diversity in bee (Hymenoptera: Apoidea) and social wasp (Hymenoptera:
Vespidae, Poistinae) community in "Campos Rupestres", Bahia, Brasil. Neotropical Entomology 35:165-174. http://dx.doi.org/ http://dx.doi.org/10.1590/S1519-566X2006000200003

Skarpe, C. 1996. Plant functional types and climatic in a southern African savanna. Journal of Vegetation Science 7:397404. http://dx.doi.org/10.2307/3236283

Thompson, K., O. L. Petchey, A. P. Askew, N. P. Dunnett, A. P. Beckman, and A. J. Willis. 2010. Little evidence for limiting similarity in a long-term study of a roadside plant community. Journal of Ecology 98:480-487. http://dx.doi.org/10.1111/j.13652745.2009.01610.x

TILMAN, D. 2001. Functional diversity. Encyclopedia of diversity 3:109-121.

Trindade-Filho, J., F. L. Sobral, M. V. Cianciaruso, and R. D. Loyola. 2012. Using indicator groups to represent bird phylogenetic and functional diversity. Biological Conservation 146:155-162. http://dx.doi.org/10.1016/j.biocon.2011.12.004

Van der Linden, P., J. Patrício, A. Marchini, N. Cid, J. M. Neto, J. C. Marques. 2012. A biological trait approach to assess the functional composition of subtidal benthic communities in an estuarine ecosystem. Ecological Indicators 20:121-133. http:// dx.doi.org/10.1016/j.ecolind.2012.02.004

Vandewalle, M., F. de Bello, M. P. Berg, T. Bolger, S. Dolédec, F. Dubs, C. K. Feld, R. Harrington, P. A. Harrison, S. Lavorel, P. M. Silva, M. Moretti, J. Niemela, P. Santos, T. Sattler, J. P. Sousa, M. T. Sykes, A. J. Vanbergen, and B. A. Woodcok. 2010. Functional traits as indicators of biodiversity response to land use changes across ecosystems and organisms. Biodiversity and Conservation 19:29212947. http://dx.doi.org/10.1007/s10531-010-9798-9

Villéger, S., G. Grenouillet, and S. Brosse. 2013. Decomposing functional â-diversity reveals that low functional â-diversity is driven by low functional turnover in European fish assemblages. Global Ecology and Biogeography 22:671-681. http:/ /dx.doi.org/10.1111/geb.12021

Violle, C., M.-L. Navas, D. Vile, E. Kazakou, C. Fortunel, I. Hummel, and E. Garnier. 2007. Let the concept of trait be functional! Oikos 116:882-892. http://dx.doi.org/10.1111/ j.2007.0030-1299.15559.x

Walker, B., A. Kinzig, and J. LangridgeJ. 1999. Plant attribute diversity, resilience and ecosystem function: the nature and significance of dominant and minor species. Ecosystems 2:95-113.

Warse, N. M. 2006. Specialization and generalization in plant-pollinator interactions: a historical perspective. Chapter 1. Pages 3-17 in: N. M. Warse, and J. Ollerton, editors. PlantPollinator Interactions - From Specialization to Generalization. The University of Chicago Press. Chicago. USA.

Willians, N. M..; E. E. Crone, T. H. Roulston, R. L. Minckley, L. Parker, and S. G. Potts. 2010. Ecological and lifehistory traits predict bee species responses to environmental disturbances. Biological Conservation 143:2280-2291. http:// dx.doi.org/10.1016/j.biocon.2010.03.024

Whittaker, R. H. 1951. A Criticism of the Plant Association and Climatic Climax Concepts. Northwest Science 25: 17-31.

Zayed, A., L. Packer, J. C. Grixti, L. Ruz, R. E. Owen, and H. Toro. 2005. Increased Genetic Differentiation in a Specialist versus a Generalist Bee: Implications for Conservation. Conservation Genetics 6:1017-1026. http://dx.doi.org/10.1007/ s10592-005-9094-5 